\title{
The survival difference between gastric cancer patients from the UK and Japan remains after weighted propensity score analysis considering all background factors
}

\author{
Takanobu Yamada · Takaki Yoshikawa - Masataka Taguri • \\ Tsutomu Hayashi · Toru Aoyama · Henry M. Sue-Ling • \\ Kiran Bonam · Jeremy D. Hayden · Heike I. Grabsch
}

Received: 9 October 2014/ Accepted: 24 February 2015/Published online: 12 March 2015

(C) The International Gastric Cancer Association and The Japanese Gastric Cancer Association 2015

\begin{abstract}
Background Previous studies comparing survival between gastric cancer (GC) patients from the West and the East were based on the assumption that background factors and prognostic factors were identical. The aim of the current study was to compare the survival of GC patients from the UK and Japan using weighted propensity score analysis after identifying all different background factors.

Methods Data from 464 patients from the Leeds Teaching Hospital NHS Trust, Leeds, UK (LTHT), and 465
\end{abstract}

T. Yamada and T. Yoshikawa contributed equally.

T. Yamada $\cdot$ T. Yoshikawa $(\bowtie) \cdot$ T. Hayashi · T. Aoyama Department of Gastrointestinal Surgery, Kanagawa Cancer Center, 2-3-2 Nakao, Asahi-ku, Yokohama, Kanagawa, Japan e-mail: yoshikawat@kcch.jp

\section{Taguri}

Department of Biostatistics and Epidemiology, Graduate School of Medicine, Yokohama City University, 4-57 Urafune,

MInami-ku, Yokohama, Kanagawa, Japan

H. M. Sue-Ling · J. D. Hayden

Department of Upper Gastrointestinal Surgery, Leeds Teaching Hospital NHS Trust, St James's Institute of Oncology,

Bexley Wing, St James's University Hospital, Leeds, UK

\section{K. Bonam}

Department of Radiology, Newcastle upon Tyne Hospitals NHS Trust, Newcastle, UK

\section{K. Bonam}

Section of Pathology and Tumor Biology, Leeds Institute of Cancer and Pathology, University of Leeds, Leeds, UK

\section{H. I. Grabsch ( ()}

Department of Pathology, Maastricht University Medical Center, P. Debyelaan 25, 6229 HX Maastricht, The Netherlands e-mail: H.Grabsch@maastrichtuniversity.nl patients from the Kanagawa Cancer Center Hospital, Yokohama, Japan $(\mathrm{KCCH})$, who had surgery for GC were analyzed. Prognostic factors for overall survival (OS) and cancer-specific survival (CSS) were identified by univariate and multivariate analyses. Survival was compared by propensity score weighting after adjusting for all significantly different background factors.

Results Most background factors were different between LTHT and KCCH patients. Unadjusted stage-specific OS and CSS were significantly better in $\mathrm{KCCH}$. Independent prognostic factors for unadjusted OS and CSS were pT and $\mathrm{pN}$ in $\mathrm{KCCH}$ and in addition tumor location, pancreatectomy, resection margin status and number of examined lymph nodes in LTHT. Even after adjusting for all background characteristics, survival remained better in $\mathrm{KCCH}$.

Conclusions These results suggest that differences in background factors are unable to fully explain the survival difference of GC patients between UK and Japan. Comprehensive studies into the biology of GC and/or host factors are needed to fully understand the survival difference.

Keywords Gastric cancer - Propensity score weighting · Prognosis · East versus West

\section{Introduction}

Gastric cancer (GC) is the third most common cause of cancer death in the world, and 723,027 patients are estimated to have died from this disease in 2012 [1]. Recent phase III clinical trials have demonstrated that multimodality treatment significantly improves the survival of locally advanced GC compared with surgery alone [2-5]. However, the type of multimodal treatment varies between countries and consists of adjuvant S-1 chemotherapy in 
Japan [2], adjuvant chemotherapy with capecitabine plus oxaliplatin in South Korea [3], adjuvant chemoradiation in the USA [4], and perioperative chemotherapy with epirubicin, cisplatin and 5-fluorouracil in the UK [5]. Furthermore, recent studies have shown that 5-year overall survival of GC patients treated by surgery alone was $60 \%$ in Japan and Korea, but only $20 \%$ in the USA and the UK [2-5].

Previous cohort studies have highlighted the survival difference in patients with resectable GC between Western and Eastern countries [6-14]. Noguchi et al. [6] reported a survival difference between high-volume centers in the USA and Japan that was no longer apparent after adjusting for tumor location. Verdecchia et al. [7] demonstrated that the survival of Italian GC patients was inferior to that of Japanese GC patients and that this survival difference disappeared after adjusting for stage. Bollschweiler et al. [8] compared the survival of Japanese and German GC patients and concluded that the country itself was a prognostic factor. Strong et al. [9] calculated the diseasespecific survival of GC patients by considering the risk of individual patients using a nomogram and demonstrated that the survival probability was different between a Korean and US high-volume center.

All previous studies comparing GC between West and East were either based on the assumption that background factors and prognostic factors were identical or considered only a limited number of background factors. As ethnicity, epidemiology and treatment are all different between the West and the East, background factors and prognostic factors are most likely also different. However, no previous study compared the survival adjusted for significantly different background factors between GC patients from the West and the East. Theoretically, three methods could be used when comparing adjusted survival comprehensively. The first one is multivariate Cox's proportional analysis [15], the second one is propensity score matching [16], and the third one is propensity score weighting [17]. The first one is not applicable when the prognostic factor and a baseline hazard function are different between the cohorts. The second one causes statistical under-power by reduction of the sample size for matching. Thus, the third one could be considered the most promising method for this type of analysis.

The aims of the current study were (1) to identify the clinicopathological background factors that are different between gastric cancer patients from the UK and Japan, (2) to identify and compare prognostic factors in both cohorts and (3) to compare survival between UK and Japan with and without adjusting for all background factors found to be significantly different by weighted propensity score analysis in order to control for differences in confounding observational factors [18].

\section{Materials and methods}

Patients from the Leeds Teaching Hospitals NHS Trust (LTHT), Leeds, UK

Patients with histologically confirmed resectable adenocarcinoma of the stomach treated by total or partial gastrectomy with macroscopically complete resection of the tumor after 1989 were selected from the database. The year 1989 was chosen to avoid a significant time bias between the two cohorts. Patients with evidence of peritoneal tumor deposits or liver metastases noted at the time of gastrectomy that were amenable to surgical resection and those with microscopic residual disease at the longitudinal resection margin (pR1) were included. However, patients with cancer of the gastro-esophageal junction and those who were lost during follow-up or received any preor postoperative chemotherapy were excluded. In total, 591 patients had surgery for gastric adenocarcinoma in Leeds between 1990 and 2009, of which 464 patients fulfilled the above inclusion criteria for the study. The median follow-up time was 20 months, ranging from 0 to 188 months.

\section{Patients from the Kanagawa Cancer Center Hospital} $(\mathrm{KCCH})$, Yokohama, Japan

To minimize the potential time bias between LTHT and $\mathrm{KCCH}$ and to exclude $\mathrm{KCCH}$ patients who received any pre- or postoperative chemotherapy, $\mathrm{KCCH}$ patients who had surgery between January 2000 and February 2004 were selected based on the same criteria as described. During this period, 506 patients had surgery for gastric adenocarcinoma in $\mathrm{KCCH}$. Thirty-five patients were excluded because of the presence of unresectable distant metastases, one patient because of neoadjuvant chemotherapy and five patients as they received postoperative chemotherapy. Thus, a total of 465 patients fulfilled the above inclusion criteria for the study in $\mathrm{KCCH}$. The median follow-up time was 63 months, ranging from 1 to 110 months.

\section{Clinical data}

Patient characteristics, clinical, surgical and pathological data were retrieved from hospital records in each institution. The 7th edition of the UICC TNM classification was used for tumor staging [19]. The survival data were obtained from outpatient clinics, the Kanagawa Prefectural registry system, and the Northern and Yorkshire Cancer Registry. This study was approved by the Local Ethics Research Committee in each institution. 
Statistical analyses

Patients' characteristics (age, gender, tumor location, extent of surgery, splenectomy, pancreatectomy, depth of invasion ( $\mathrm{pT}$ category), lymph node status ( $\mathrm{pN}$ category), absence or presence of distant metastases ( $\mathrm{M}$ category), pTNM stage, absence or presence of tumor at the resection margin ( $\mathrm{R}$ category), number of examined lymph nodes and histological tumor type according to the Lauren classification [20]) were compared using the chi-square test. Overall survival (OS) was defined as time from surgery to date of death due to any cause or date of last follow-up. Cancer-specific survival (CSS) was defined as time from surgery to date of death due to cancer or date of last followup/death due to other cause. The survival probability of both institutions was calculated by the Kaplan-Meier method and compared by log-rank test. To identify significant prognosticators for patients from each institution, uni- and multivariate analyses were performed using a Cox regression proportional hazard model. The differences in the background clinicopathological characteristics between patients from both institutions were adjusted through propensity score weighting [18]. The propensity score for each patient was estimated by a logistic regression model using institution (LTHT or $\mathrm{KCCH}$ ) as dependent variable and all patient characteristics as independent variables. Next, adjusted survival probabilities were calculated by the Kaplan-Meier method and compared by log-rank test using the sandwich variance estimator [21]. The unadjusted and propensity score adjusted $\log$ HRs (logHRs) were compared using a $Z$ test. The standard error of the difference in $\operatorname{logHRs}$ was calculated using the robust variance estimator, which takes the correlation of $\operatorname{logHRs}$ as well as propensity score weighting into account [22]. $p$ values $<0.05$ were considered to be significant. All data analyses were carried out using SPSS version 20 for Windows (SPSS Inc., Chicago, IL, USA) or SAS version 9.3 for Windows (SAS Institute Inc, Cary, NC, USA).

\section{Results}

All unadjusted clinical and pathological factors except tumor location were significantly different between $\mathrm{KCCH}$ and LTHT (Table 1). KCCH patients were significantly younger and had a significantly higher proportion of males. Total gastrectomy was the predominant type of surgery in LTHT and splenectomy and/or pancreatectomy were more frequently performed in LTHT patients compared to $\mathrm{KCCH}$ patients. Tumor invasion was deeper and lymph node metastases were more frequent in LTHT patients; $52 \%$ of $\mathrm{KCCH}$ patients had pT1 cancers and $60 \%$ had pN0 disease, while $51 \%$ of LTHT patients had pT4 cancers and $70 \%$ had at least one lymph node metastasis. Distant metastasis (M) was more frequent in $\mathrm{KCCH}$ than LTHT patients. The number of examined lymph nodes was significantly higher in $\mathrm{KCCH}$ patients; $92 \%$ of $\mathrm{KCCH}$ patients had more than 15 examined lymph nodes compared to $61 \%$ of LTHT patients. LTHT patients had more frequently a more advanced pTNM stage. Thus, stage I disease was observed in $57 \% \mathrm{KCCH}$ patients, but only in $14 \%$ LTHT patients, while stage III disease was present in $22 \% \mathrm{KCCH}$ patients and in $55 \%$ LTHT patients. The histological tumor type was also significantly different with diffuse type GC being more frequently seen in $\mathrm{KCCH}$ patients than in LTHT patients.

Identification of prognostic factors and comparative survival analyses without adjusting for background factors

For $\mathrm{KCCH}$ patients, age, tumor location, $\mathrm{pT}$ and $\mathrm{pN}$ were found to be independent prognostic factors for OS and CSS and distant metastases (M) for CSS. For LTHT patients, pancreatectomy, resection margin status and the number of examined lymph nodes were independent prognostic factors for OS and CSS in addition to the prognostic factors identified in $\mathrm{KCCH}$ patients. Results from survival analyses are detailed in Tables 2 and 3. Next, we examined independent prognostic factors for OS and CSS in LTHT patients with number of nodes greater than 15 to exclude any potential effect of stage migration. The number of examined lymph nodes was still an independent prognostic factor for OS [HR (95\% CI) $0.986 \quad(0.974-0.998)$, $p=0.028]$ and CSS [HR (95\% CI) 0.980 (0.965-0.995), $p=0.010]$.

The 5-year OS and CSS of $\mathrm{KCCH}$ patients were significantly better. The 5-year OS was $76.6 \%$ in $\mathrm{KCCH}$ patients compared to $31.5 \%$ in LTHT patients [HR $(95 \%$ CI) 4.381 (3.553-5.402), $p<0.0001]$. The 5-year CSS was $81.4 \%$ in $\mathrm{KCCH}$ patients compared with $45.3 \%$ in LTHT patients [HR (95\% CI) 3.869 (3.028-4.944), $p<0.0001$ ] (Fig. 1a, b).

Comparative survival analyses after adjusting for background factors

For the survival analyses, patient numbers in each background factor were adjusted by weighting propensity score. After this adjustment, the clinical and pathological factors were no longer different between LTHT and KCCH (Table 4). The difference in survival probabilities was significantly reduced between the two cohorts $(p<0.0001)$. However, the 5-year adjusted OS and the 5 -year adjusted CSS remained significantly better in $\mathrm{KCCH}$ patients than in LTHT patients. The 5-year adjusted OS 
Table 1 Comparison of unadjusted background characteristics between $\mathrm{KCCH}$ and LTHT
Bold values are statistically significant

$\mathrm{KCCH}$ Kanagawa Cancer Center Hospital, LTHT Leeds Teaching Hospital NHS Trust
$\mathrm{KCCH}(N=465)$

No. $(\%)$

LTHT $(N=464)$

No. $(\%)$

Age

$\geq 65$

$<65$

Gender

Male

Female

Location

Lower/middle third

Upper third

Entire stomach

Surgery type

Local/distal resection

Total/proximal gastrectomy

Splenectomy

Yes

No

Pancreatectomy

Yes

No

$\mathrm{T}$ categories

T1

$\mathrm{T} 2$

T3

T4

$\mathrm{N}$ categories

N0

N1

$\mathrm{N} 2$

N3

$\mathrm{M}$ categories

M0

M1

Stage (UICC TNM 7th)

I

II

III

IV

Tumor at resection margin

R0

R1

No. of examined lymph nodes

$<15$

$\geq 15$

Lauren classification

Diffuse type

Intestinal type

Mixed type
$195(42)$

$270(58)$

341 (73)

124 (27)

344 (74)

$103(22)$

18 (4)

$295(63)$

$170(37)$

$36(8)$

429 (92)

5 (1)

460 (99)

$244(52)$

57 (12)

24 (5)

140 (30)

$278(60)$

$50(11)$

46 (10)

91 (20)

425 (91)

40 (9)

263 (57)

66 (14)

102 (22)

34 (7)

441 (95)

24 (5)

38 (8)

427 (92)

$250(54)$

185 (40)

286 (62)

30 (6)
67 (14)

180 (39)

284 (61)

105 (23)

$<0.0001$

0.0001

0.160

27 (27)

209 (45)

$<0.0001$

87 (19)

$<0.0001$

43 (9)

$<0.0001$

421 (91)

50 (11)

41 (9)

138 (30)

235 (51)

142 (31)

88 (19)

97 (21)

137 (30)

451 (97)

13 (3)

66 (14)

132 (28)

253 (55)

13 (3)

$<0.0001$

0.0001

$<0.0001$

73 (16)
$<0.0001$

$<0.0001$ 


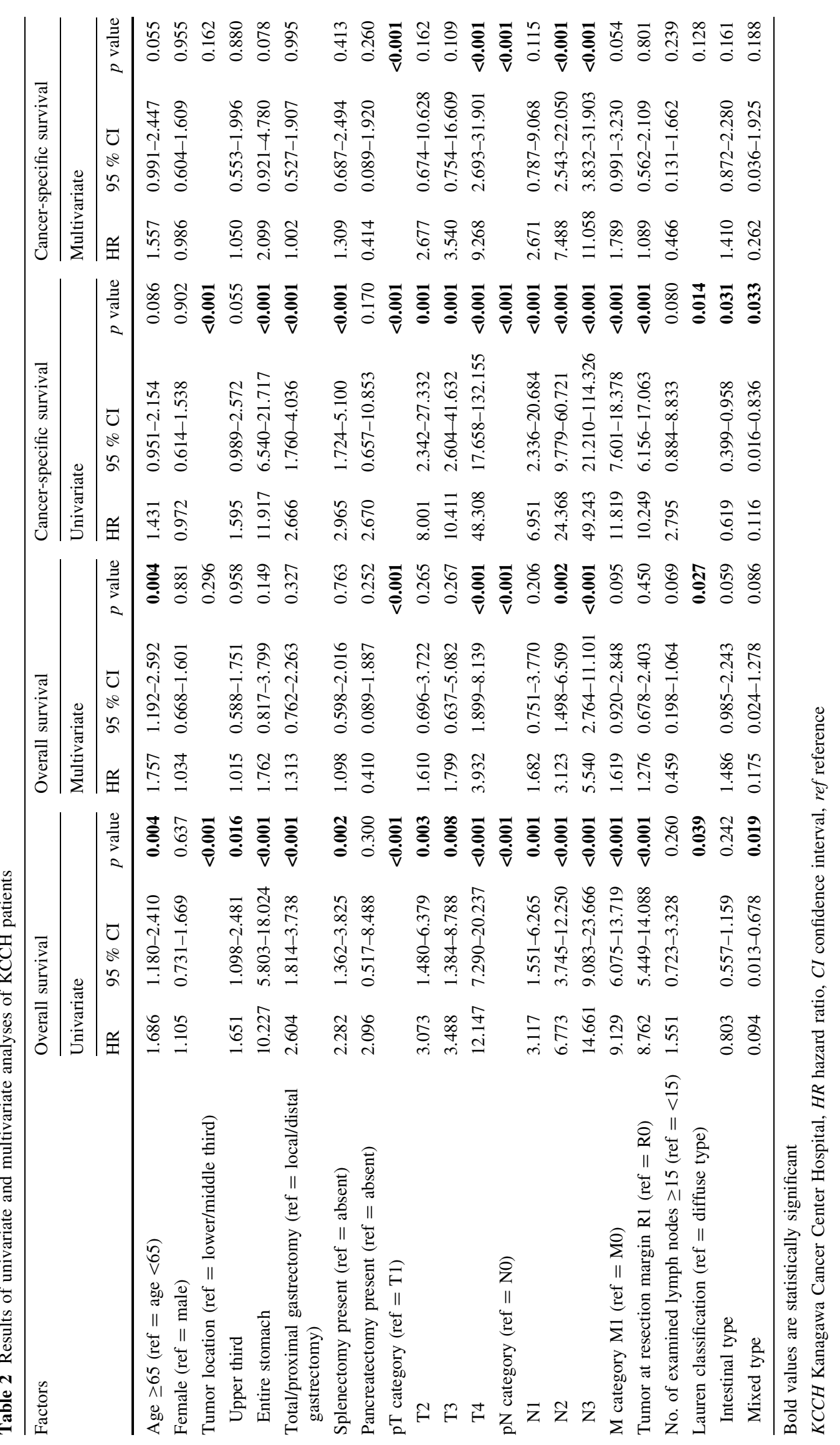




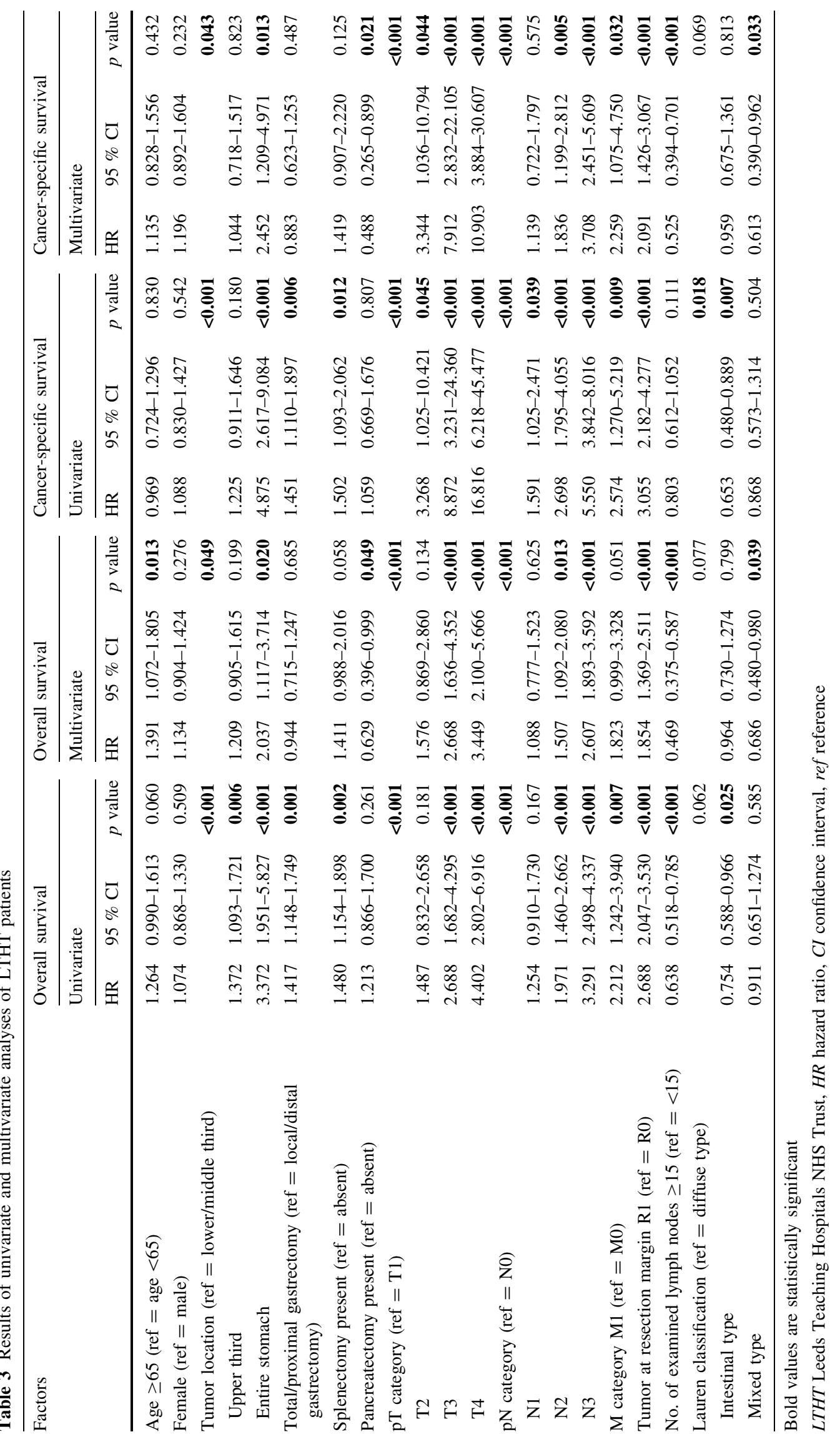




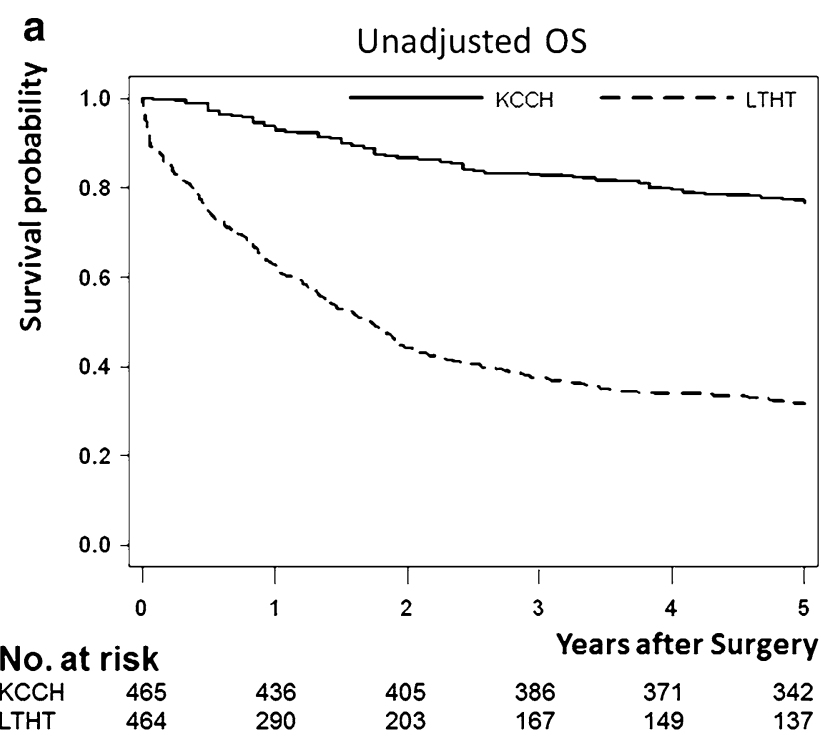

Fig. 1 a Unadjusted overall survival (OS) in KCCH and LTHT. The 5-year unadjusted OS was $76.6 \%$ in $\mathrm{KCCH}$ patients compared with $31.5 \%$ in LTHT patients [HR (95\% CI) 4.381 (3.553-5.402), $p<0.0001]$. b Unadjusted cancer-specific survival (CSS) in $\mathrm{KCCH}$

was $69.0 \%$ in $\mathrm{KCCH}$ patients compared to $52.2 \%$ in LTHT patients [HR (95\% CI) 1.832 (1.487-2.257), $p<0.0001]$. The 5-year CSS was $75.3 \%$ in $\mathrm{KCCH}$ patients compared to $64.9 \%$ in LTHT patients [HR (95\% CI) 1.590 (1.239-2.039), $p=0.0003$ ] (Fig. 2a, b).

\section{Discussion}

Some investigators have reported that the survival of gastric cancer (GC) patients was different between the West and the East, whereas others reported the opposite [6-14]. However, most studies only considered selected factors when analyzing their data sets. This is the first study to compare the survival between GC patients from the UK and Japan after adjusting for all available background differences using propensity score weighting.

The present study demonstrates that prognostic factors of GC patients are different in the West and the East and, in particular, that there are more clinicopathological factors related to prognosis in the West compared to the East. As expected, the universally accepted prognostic factors $\mathrm{pT}$ and $\mathrm{pN}$ were identified as independent prognosticators in both cohorts. The results from our study also support the previously described finding that patient age at the time of diagnosis is significantly associated with overall survival in Western and Eastern GC patients [23, 24]. Confirming previous reports [25-27], tumor size was also identified as a poor prognostic factor in the current study.

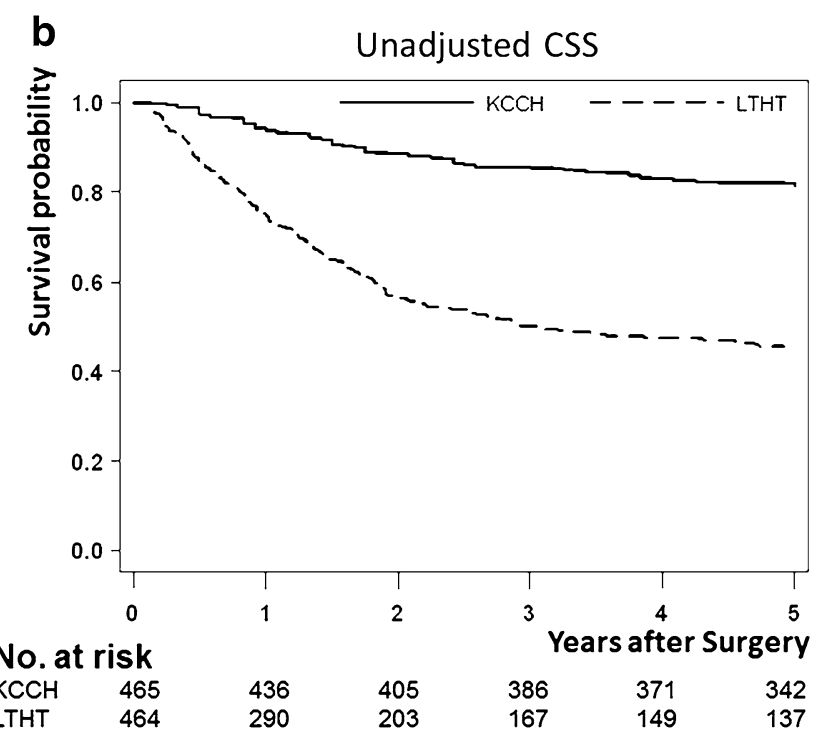

and LTHT. The 5-year unadjusted CSS was $81.4 \%$ in $\mathrm{KCCH}$ patients compared with $45.3 \%$ in LTHT patients [HR (95\% CI) 3.869 (3.028-4.944), $p<0.0001]$

Pancreatectomy and resection margin status were significant prognosticators in LTHT patients only. This result could simply be related to the higher frequency of total gastrectomy with pancreatectomy in LTHT patients and the relative low frequency of $\mathrm{R} 1$ resections in $\mathrm{KCCH}$ patients. A previous study demonstrated that the prognosis of the patients treated by a total gastrectomy was poorer compared to those who were treated by distal gastrectomy [28]. Resection margin status has been reported to have prognostic value in previous studies comparing GC patients from the West and the East [29, 30].

The total number of examined lymph nodes was only a significant prognosticator in LTHT patients. This finding is consistent with results from the SEER database analyzing data from US (e.g., Western) GC patients, which showed that the overall survival of GC patients was highly dependent on the total number of examined lymph nodes in every disease stage [31]. In contrast, a recent study in GC from Taiwan (e.g., from the East) reported that the total number of examined nodes was not related to survival in patients with T1 disease [32], supporting the lack of prognostic value of the number of examined nodes in $\mathrm{KCCH}$ patients as $52 \%$ of $\mathrm{KCCH}$ patients had $\mathrm{T} 1$ disease opposed to $11 \%$ of LTHT patients. However, one also needs to consider that a relatively low total number of examined lymph nodes may result in an inaccurately low nodal stage, e.g., stage migration. As $39 \%$ of LTHT patients but only $8 \%$ of $\mathrm{KCCH}$ patients had less than 15 lymph nodes examined, stage migration and the prognostic effect of the number of examined lymph nodes would only 
Table 4 Comparison of propensity score-adjusted background characteristics between $\mathrm{KCCH}$ and LTHT
$\mathrm{KCCH}$ Kanagawa Cancer Center Hospital, LTHT Leeds Teaching Hospital NHS Trust
$\mathrm{KCCH}(N=465)$

No. $(\%)$

LTHT $(N=464)$

No. $(\%)$

Age

$\geq 65$

$<65$

$250.3(54)$

214.7 (46)

$262.3(57)$

0.706

Gender

Male

$337.2(73)$

0.887

Female

127.8 (27)

$339.5(73)$

124.5 (27)

Location

Lower/middle third

$328.1(71)$

348.7 (75)

0.576

Upper third

119.2 (26)

$102.3(22)$

Entire stomach

$17.8(4)$

$13.0(3)$

Surgery type

Local/distal resection

$260.3(56)$

$279.6(60)$

0.475

Total/proximal gastrectomy

204.7 (44)

$184.5(40)$

Splenectomy

Yes

$52.3(11)$

$56.7(12)$

0.754

412.7 (89)

$407.3(88)$

Pancreatectomy

Yes

$10.3(2)$

$454.7(98)$

$21.5(5)$

0.204

No

$\mathrm{T}$ categories

T1

$\mathrm{T} 2$

T3

T4

$\mathrm{N}$ categories

N0

N1

$\mathrm{N} 2$

N3

$\mathrm{M}$ categories

M0

M1

Stage (UICC TNM 7th)

I

II

III

IV

Tumor at resection margin

R0

$67.0(36)$

$55.6(12)$

$59.1(13)$

$183.3(39)$

217.9 (47)

$94.7(20)$

$61.0(13)$

$91.4(20)$

436.5 (94)

$28.5(6)$

$189.5(41)$

84.8 (18)

$165.6(36)$

$25.1(5)$

$439.8(95)$

$25.2(5)$

No. of examined lymph nodes

$<15$

$71.6(15)$

$393.4(85)$

$\geq 15$

Lauren classification

Diffuse type

203.7 (44)

$231.0(50)$

$30.3(7)$
$442.5(95)$

0.383

$40.0(9)$

$78.0(17)$

$168.0(36)$

$191.5(41)$

0.526

$77.2(17)$

$104.3(22)$

$91.0(20)$

$445.0(96)$

0.285

19.0 (4)

$156.3(34)$

0.519

126.0 (27)

$162.8(35)$

$19.0(4)$

$426.4(92)$

0.168

$37.6(8)$

100.5 (22)

0.210

363.5 (78)

170.6 (37)

0.396
178.0 (38)

Mixed type

250.9 (54)

$42.6(9)$ 


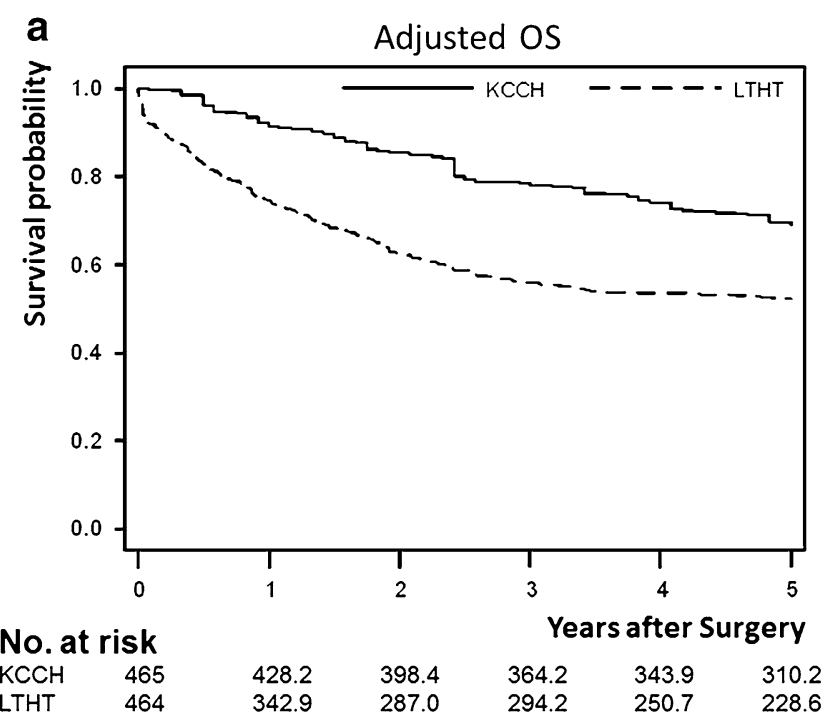

Fig. 2 a Adjusted overall survival (OS) in KCCH and LTHT. The 5 -year adjusted OS was $69.0 \%$ in $\mathrm{KCCH}$ patients compared with $52.2 \%$ in LTHT patients [HR (95\% CI) 1.832 (1.487-2.257), $p<0.0001]$. b Adjusted cancer-specific survival (CSS) in $\mathrm{KCCH}$ and

become evident in LTHT patients. However, when we restricted our analysis to LTHT patients with more than 15 investigated nodes, the number of nodes remained an independent prognostic factor. The relatively low total number of examined lymph nodes in LTHT patients could be an indicator of a less extended D1-type lymph node dissection by the LTHT surgeons or reflect some unknown biological factor. Recently published results from the longterm follow-up of the Dutch trial comparing survival after D2 versus D1 resection showed that disease-specific survival was significantly longer in patients who had a D2 resection [33].

The current study shows that the survival between GC patients from the UK and Japan remains different even after adjustment for all significantly different clinicopathological factors although the difference was reduced by adjustment. Our results are in contrast to those published previously, which reported that differences in selected background factors are responsible for the survival difference between GC patients in the West and the East [6-14]. However, our study is the first to compare the survival adjusted by all background factors using propensity score weighting. Weighted propensity score analysis as used in the current study has been shown to perform better than logistic regression analysis in controlling for confounders if many baseline characteristics need to be included in the regression model [34]. Previous studies performed their statistical analyses based on the assumption that prognosticators and/or background factors were identical between the different cohorts. Our study clearly demonstrated that some of the prognosticators and most of

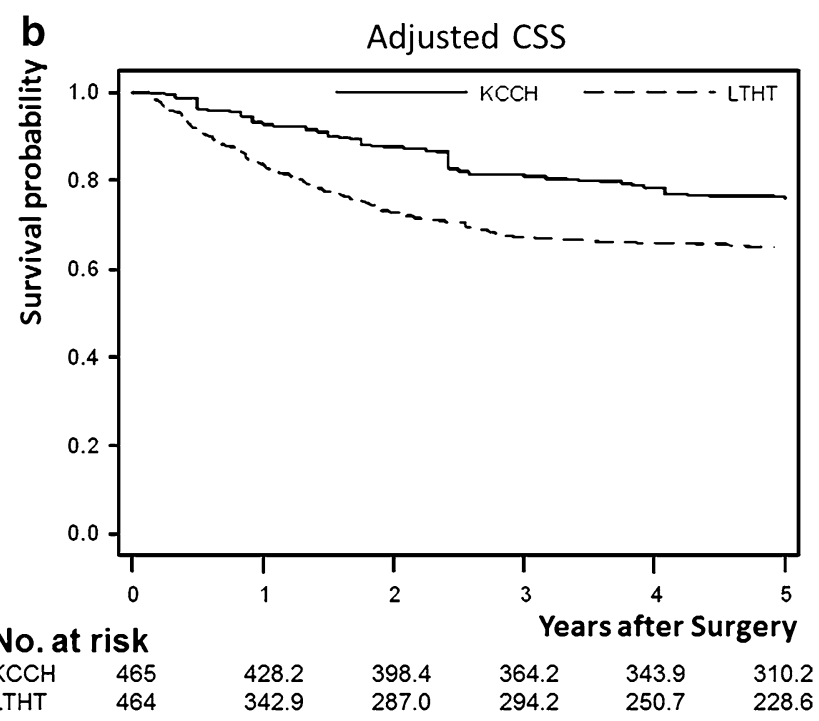

LTHT. The 5-year CSS was $75.3 \%$ in $\mathrm{KCCH}$ patients compared with $64.9 \%$ in LTHT patients [HR (95\% CI) 1.590 (1.239-2.039), $p=0.003]$

the background factors were different between cohorts. Most interestingly, our study showed that although the survival difference between cohorts became smaller after adjustment for all different background factors, a significant survival difference remained between the two cohorts. This may suggest that there are some unknown factors such as a difference in cancer biology or host factors.

The present study has some limitations. This was a retrospective observational study comparing data from two different hospitals vulnerable to confounding by case mix despite using the same inclusion and exclusion criteria. Information about the intended extent of lymph node dissection (D1 versus D2), patient performance status, existing comorbidities at the time of surgery and postoperative complications, as well as treatment modality at the time of disease recurrence, was not available and could therefore not be compared between the cohorts. Thus, the adjusted survival difference in this study could potentially be related to the difference in extent of lymph node dissection or to background factors we were unable to analyze and compare. In this study, the difference at 5 years was $16.8 \%$ for adjusted overall survival and $10.4 \%$ for adjusted cancerspecific survival between the two cohorts. One may argue that this difference could simply reflect the difference in the extent of nodal dissection between the two cohorts. However, a Dutch phase III study comparing D1 and D2 demonstrated that the difference at 15 years was $8 \%$ for overall survival and $11 \%$ for gastric-cancer-related death rate [33]. In their study, overall 15-year survival was $21 \%$ for D1 and $29 \%$ for D2. On the other hand, a Taiwanese 
phase III trial showed that the difference at 5 years was $5.9 \%$ for overall survival and $5.3 \%$ for disease-specific survival [35]. In that study, the overall 5-year survival was $53.6 \%$ for D1 and $59.5 \%$ for D3 (corresponding to the current D2). In the present study, 5-year adjusted overall survival was $52.2 \%$ for LTHT and $69.0 \%$ for $\mathrm{KCCH}$, which is close to that reported in the Taiwanese phase III trial. One could therefore conclude that the expected difference due to D2 surgery in the current study would most likely be more similar to the Taiwanese trial, e.g., between 5 and $6 \%$. Hence, the survival difference between the LTHT and KCCH cohort after adjustment for all available background factors cannot be fully explained by a difference in nodal dissection. Furthermore, the biological behavior of different histology types could be different depending on the disease stage, which could not be adjusted by propensity-score weighting [36].

Due to the lower incidence of GC in the UK, the LTHT cohort included patients between the 1990s and 2000s, while the $\mathrm{KCCH}$ cohort only included patients from 2000 onwards. Although time bias between LTHT and $\mathrm{KCCH}$ is minimal, differences in surgical treatment, clinical and pathological staging by treatment period could well be present. While the propensity scores used in the adjusted survival analyses can account for distribution biases within variables, weighted propensity score analysis cannot take into account unknown confounding factors. Thus, for example, the difference in the distribution of the $\mathrm{pN}$ category can be adjusted by propensity score weighting, but stage migration cannot be adjusted for as it is not known whether there is stage migration or not.

In summary, many clinicopathological background factors and some significant prognosticators were found to be different between patients with resectable gastric cancer from Japan and the UK. A significant survival difference between the two cohorts was still evident after adjusting for these background factors using weighted propensity score analysis. Our results suggest that comparative studies into gastric cancer biology and/or host factors are needed to fully understand the survival difference between gastric cancer patients from the West and the East.

Acknowledgments This work was supported by grants from the non-profit organization Kanagawa Standard Anti-cancer Therapy Support System (Yokohama, Japan).

Conflict of interest None.

\section{References}

1. Ferlay J, Soerjomataram I, Ervik M, et al. GLOBOCAN 2012 v1.0, Cancer Incidence and Mortality Worldwide: IARC CancerBase No. 11 [Internet]. Lyon, France: International Agency for
Research on Cancer; 2013. Available at: http://globocan.iarc.fr. Accessed March 5, 2014.

2. Sakuramoto S, Sasako M, Yamaguchi T, et al. Adjuvant chemotherapy for gastric cancer with S-1, an oral fluoropyrimidine. N Engl J Med. 2007;357:1810-20.

3. Bang YJ, Kim YW, Yang HK, et al. Adjuvant capecitabine and oxaliplatin for gastric cancer after D2 gastrectomy (CLASSIC): a phase 3 open-label, randomised controlled trial. Lancet. 2012;379:315-21.

4. Macdonald JS, Smalley SR, Benedetti J, et al. Chemoradiotherapy after surgery compared with surgery alone for adenocarcinoma of the stomach or gastroesophageal junction. N Engl J Med. 2001;345:725-30.

5. Cunningham D, Allum WH, Stenning SP, et al. Perioperative chemotherapy versus surgery alone for resectable gastroesophageal cancer. N Engl J Med. 2006;355:11-20.

6. Noguchi Y, Yoshikawa T, Tsuburaya A, et al. Is gastric carcinoma different between Japan and the United States? Cancer. 2000;89:2237-46.

7. Verdecchia A, Mariotto A, Gatta G, et al. Comparison of stomach cancer incidence and survival in four continents. Eur $\mathrm{J}$ Cancer. 2003;39:1603-9.

8. Bollschweiler E, Boettcher K, Hoelscher AH, et al. Is the prognosis for Japanese and German patients with gastric cancer really different? Cancer. 1993;71:2918-25.

9. Strong VE, Song KY, Park $\mathrm{CH}$, et al. Comparison of gastric cancer survival following R0 resection in the United States and Korea using an internationally validated nomogram. Ann Surg. 2010;251:640-6.

10. Noguchi Y, Imada T, Matsumoto A, et al. Radical surgery for gastric cancer -review of the Japanese literature. Cancer. 1989;64:2053-62.

11. Ohtsu A, Yoshida S, Saijo N. Disparities in gastric cancer chemotherapy between the East and West. J Clin Oncol. 2006;24:2188-96.

12. Hsu C, Shen YC, Cheng CC, et al. Geographic difference in safety and efficacy of systemic chemotherapy for advanced gastric or gastroesophageal carcinoma: a meta-analysis and metaregression. Gastric Cancer. 2012;15:265-80.

13. Maruyama M, Takeshita K, Endo M, et al. Clinicopathological study of gastric carcinoma in high- and low-mortality countries: comparison between Japan and the United States. Gastric Cancer. 1998;1:64-70.

14. Davis PA, Sano T. The difference in gastric cancer between Japan, USA, and Europe: What are the facts? What are the suggestions? Oncol Hematol. 2001;40:77-94.

15. Chang IM, Gelman R, Pagano M. Corrected group prognostic curves and summary statistics. J Chronic Dis. 1982;35(8):669-74.

16. D'Agostino RB Jr. Propensity score methods for bias reduction in the comparison of a treatment to a non-randomized control group. Stat Med. 1998;17(19):2265-81.

17. Hirano K, Imbens GW. Estimation of causal effects using propensity score weighting: an application to data on right heart catheterization. Health Serv Outcomes Res Method. 2001;2: 259-78.

18. Cole SR, Hernán MA. Adjusted survival curves with inverse probability weights. Comput Methods Programs Biomed. 2004;75:45-9.

19. Sobin LH, Gospodarowicz MK, Witterkind $\mathrm{CH}$. International Union Against Cancer (UICC) TNM Classification of Malignant Tumors. 7th ed. Oxford: Wiley-Blackwell; 2009.

20. Lauren T. The two histologic main types of gastric carcinoma. Acta Pathol Microbiol Scand. 1965;64:31-49.

21. Lin DY, Wei LJ. The robust inference for the proportional hazards model. J Am Stat Assoc. 1989;84:1074-8. 
22. Lin DY. Cox regression analysis of multivariate failure time data: the marginal approach. Stat Med. 1994;13:2233-47.

23. Kattan MW, Karpeh MS, Mazumdar M, et al. Postoperative nomogram for disease-specific survival after an R0 resection for gastric carcinoma. J Clin Oncol. 2003;21:3647-50.

24. Han DS, Suh YS, Kong SH, et al. Nomogram predicting longterm survival after $\mathrm{d} 2$ gastrectomy for gastric cancer. J Clin Oncol. 2012;30:3834-40.

25. Aoyama T, Yoshikawa T, Watanabe T, et al. Macroscopic tumor size as an independent prognostic factor for stage II/III gastric cancer patients who underwent D2 gastrectomy followed by adjuvant chemotherapy with S-1. Gastric Cancer. 2011;14:274-8.

26. Kunisaki C, Makino H, Takagawa R, et al. Tumor diameter as a prognostic factor in patients with gastric cancer. Ann Surg Oncol. 2008;15:1959-67.

27. Saito H, Osaki T, Murakami D, et al. Macroscopic tumor size as a simple prognostic indicator in patients with gastric cancer. Am J Surg. 2006;192:296-300.

28. Kunisaki C, Akiyama H, Nomura M, et al. Surgical outcomes for early gastric cancer in the upper third of the stomach. J Am Coll Surg. 2005;200:15-9.

29. Jang JH, Beron RI, Ahn HS, et al. Clinicopathological features of upper third gastric cancer during a 21-year period (single center analysis). J Gastric Cancer. 2010;10:212-8.
30. Raziee HR, Cardoso R, Seevaratnam R, et al. Systematic review of the predictors of positive margins in gastric cancer surgery and the effect on survival. Gastric Cancer. 2012;15:S116-24.

31. Smith DD, Schwarz RR, Schwarz RE. Impact of total lymph node count on staging and survival after gastrectomy for gastric cancer: data from a large US-population database. J Clin Oncol. 2005;23:7114-24.

32. Hsu JT, Lin CJ, Sung CM, et al. Prognostic significance of the number of examined lymph nodes in node-negative gastric adenocarcinoma. Eur J Surg Oncol. 2013;39:1287-93.

33. Songun I, Putter H, Kranenbarg EM, et al. Surgical treatment of gastric cancer: 15-year follow-up results of the randomised nationwide Dutch D1D2 trial. Lancet Oncol. 2010;11:439-49.

34. Cepeda MS, Boston R, Farrar JT, et al. Comparison of logistic regression versus propensity score when the number of events is low and there are multiple confounders. Am J Epidemiol. $2003 ; 158: 280-7$.

35. Wu CW, Hsiung CA, Lo SS, et al. Nodal dissection for patients with gastric cancer: a randomised controlled trial. Lancet Oncol. 2006;7:309-15.

36. Zu H, Wang H, Li C, Xue Y. Clinicopathologic characteristics and prognostic value of various histological types in advanced gastric cancer. Int J Clin Exp Pathol. 2014;7:5692-700. 